Elkahloun, Abdel G.

[55]

\section{Gene expression profile analysis of 13 human breast cancer specimens using laser capture microdissection and CDNA arrays}

\author{
Abdel G. Elkahloun ${ }^{1}$, Greg Robinson ${ }^{2} \&$ Dennis C. Sgroi ${ }^{3}$ \\ ${ }^{1}$ Cancer Genetic Branch, National Human Genome Research Institute, National \\ Institutes of Health, Bethesda, Maryland, USA \\ ${ }^{2}$ Opthalmology Research, Children's Hospital, Boston, Massachusetts, USA \\ ${ }^{3}$ Molecular Pathology Unit, Massachusetts General Hospital, Boston, \\ Massachusetts, USA
}

The advent of high-density complementary DNA microarray technology, with its capacity for simultaneous monitoring of thousands of genes, provides a unique opportunity for high-throughput expression analysis of cancer. Although most current microarray studies have been performed with genetic material obtained in vitro, a major advance in functional genomic investigations would be the ability to perform array-based expression analysis with genetic material obtained in vivo and originating from morphologically distinct cellular populations, including various stages of cancer progression. Until recently, in vivo analysis of tumor-specific genomic alterations, array-based or otherwise, has been hampered by the inability to obtain specific cell types accurately from cancerous tissue. The recent development of laser capture microdissection allows for accurate and rapid procurement of specific cell populations within complex tissue and provides the opportunity to perform array-based expression profiling of genetic material obtained in vivo. We describe the combined use of laser capture microdissection and highthroughput complementary DNA microarrays to monitor gene expression levels in 13 breast cancer specimens. By using different data visualization tools (clustering and multidimensional scaling) we demonstrate that expression profiles of more than 8,000 genes can be successfully generated using non-amplified RNA derived from distinct cell populations within several different morphological stages of human breast cancer.

English, Jessie

\section{Expression profiling of lung cancer cell lines}

Priya Dayananth ${ }^{1}$, Terri McClanahan², Ferdous Gheyas ${ }^{3}$, Marco Hernandez ${ }^{4}$, Wei Ding ${ }^{4}$, Luquan Wang ${ }^{4}$, Jonathan Greene $^{4}$, Weihong Jin ${ }^{1}$, Paul Kirschmeier ${ }^{1} \&$ Jessie English ${ }^{1}$

${ }^{1}$ Tumor Biology Department, SPRI

${ }^{2}$ DNAX Research Institute, USA

${ }^{3}$ Biostatistics Group, SPRI

${ }^{4}$ Bioinformatics Group, SPRI

We determined the molecular profiles of seven human lung cancer cell lines using commercially available large-scale DNA arrays (Incyte Genomics) composed of 60,000 elements $(=45,000$ genes). Relative gene expression was determined by comparing the expression of normal primary epithelial lung cell lines to that of three SCLC cell lines and four NSCLC cell lines. Few genes were differentially expressed when independently derived primary lung cell lines (NHBE and SAEC) were compared with each other. However, we detected substantial differences in gene expression when comparing tumor cell lines with normal cell lines. The largest gene expression changes occurred in cell-surface markers and cytoskeletal elements. Of genes with greater than eightfold differential expression in at least one cell line, over $15 \%$ were members of five gene families: cytokeratins, laminin 5, fibronectin, integrins and annexins. A hierarchical clustering algorithm was used to analyze gene expression changes (threefold or greater) across eight probe pairs. We grouped probe pairs into clusters to categorize relationships among cell lines. The three SCLC cell lines formed one cluster and the four NSCLC cell lines clustered together. The normal cell lines seemed to be distinct from both SCLC and NSCLC. Using cluster analysis of individual genes we identified a cluster containing genes involved in mitotic pathways and up-regulated in most tumor cell lines. Four genes were represented two or more times within this cluster and placed in adjacent rows. The capacity of this method of statistical analysis to group these genes within the same cluster and as adjacent records supports the validity and reproducibility of our experimental approach.

Farnham, Peggy

[57]

\section{Use of chromatin immunoprecipitation to study transcriptional deregulation in cancer cells}

Peggy Farnham ${ }^{1}$, Carrie Graveel ${ }^{1}$, Antonis Kirmizis ${ }^{1}$, Stephanie Bartley ${ }^{1}$, Alexander Kel${ }^{2}$, Olga Kel-Margoulis ${ }^{2}$, Edgar Wingender ${ }^{3}$, Michael Zhang ${ }^{4}$, Tim Jatkoe ${ }^{5}$

$\&$ Steven Madore ${ }^{5}$

${ }^{1}$ University of Wisconsin, Madison, Wisconsin, USA

${ }^{2}$ Institute of Cytology and Genetics, Novosibirsk, Russia

${ }^{3}$ Gesellschaft für Biotechnologische Forschung mbH, Braunschweig, Germany

${ }^{4}$ Cold Spring Harbor Laboratory, Cold Spring Harbor, New York, USA

${ }^{5}$ Pfizer Global Research and Development, Ann Arbor, Michigan, USA

Many human cancers are caused by deregulation of specific transcription factors. To study the mechanisms by which transcriptional deregulation causes neoplasia, we are using a chromatin immunoprecipitation protocol to complement three different types of gene expression profiling. We first generated a weight matrix based on a set of known E2F binding sites and then used this weight matrix to search the genome for potential E2F binding sites. Using the chromatin immunoprecipitation assay, we confirmed the identification of a set of new E2F target genes. As a second approach, we have used the chromatin immunoprecipitation protocol to characterize the transcription complexes bound to $\beta$-catenin target genes that were identified by others using gene expression profiling of tissue culture cells. Using primary tumor samples, we show a direct recruitment of both $\beta$ - and $\gamma$-catenin to certain cellular promoters. Finally we have used oligonucleotide microarrays and representational difference analysis to identify a set of genes that are highly upregulated in liver tumors. Our current goals are to identify common regulatory regions in the promoters that regulate these messenger RNAs specific to liver tumors and to characterize the components of the transcription complexes bound to these promoters using our chromatin immunoprecipitation protocol.

Fox, Jay

[58]

\section{Melanoma gene expression during matrix degradation and contraction} Jay Fox ${ }^{1}$, Paul Gallagher ${ }^{1}$, Bao Yongde ${ }^{1}$, Roswitha Nischt ${ }^{2} \&$
Cornelia Mauch

${ }^{1}$ University of Virginia, Charlottesville, Virginia, USA

${ }^{2}$ University of Cologne, Cologne, Germany

We have previously demonstrated that the expression of MMPs, MT-MMPs and integrins in human melanoma cell lines is differentially regulated depending on the type and assembly state of the extracellular matrix on which the cells are seeded. We 


\section{Abstracts: Session I}

examined differential gene expression over time as the human melanoma cell line VMM 5 degraded and contracted collagen lattices. We observed a correlation of ADAM 9 expression with the organizational state of the extracellular matrix. ADAM 9 is downregulated on organized matrix, as represented in our model system by growth in a collagen lattice compared with growth as a monolayer on unorganized matrix represented by gelatin or other single matrix components. This regulation is the opposite of that previously observed for MT1-MMP and MMP-9. ADAM 10, another proteolytically active reprolysin, showed a similar tendency. As the collagen lattice is contracted over time, upregulation of ADAM 10 occurs, which is the opposite of the regulation observed for MT1-MMP and MMP-2. Other proteins of interest, which were shown to be differentially expressed on the basis of matrix state, include TIMP 3, cadherin F1B1, and plasminogen activator inhibitor1. The results of this study suggest a role for ADAM 9 and ADAM 10 in the invasion and migration of melanoma with their expression dependent on the organizational state of the matrix with which the cells are interacting.

Frengen, Eirik

\section{A high-resolution integrated map of the breast cancer loss of heterozygosity region on human chromosome 16q22.1}

Eirik Frengen ${ }^{1}$, Philippe Rocca-Serra ${ }^{2}$, Sergey Shaposhnikov ${ }^{1}$, Laurence Taine ${ }^{3}$, J im Thorsen ${ }^{1}$, Azza Abd El Monéim³ ${ }^{3}$, Michel Longy ${ }^{2}$, Hans Prydz ${ }^{1} \&$ Françoise Dorion-Bonnet ${ }^{2}$

${ }^{1}$ Biotechnology Centre of Oslo, University of Oslo, Norway ${ }^{2}$ Laboratoire de Génétique Moléculaire (EA515), Institut Bergonié, Bordeaux, France

${ }^{3}$ Laboratoire de Génétique, Centre Hospitalier Universitaire Pellegrin, Bordeaux, France

Loss of heterozygosity on human chromosome 16q is frequently observed in both ductal and lobular invasive breast carcinomas. We have generated a 2.8 -megabase PAC contig covering the smallest region of loss of heterozygosity overlap on 16q22.1 (SRO2). We established the contig orientation with two-color fluorescence in situ hybridization and verified that the contig faithfully represents the $\mathrm{SRO} 2$ region using long-range mapping. We have identified 68 transcripts in the map on the basis of expressed sequence tag screening and $\mathrm{CpG}$ island subcloning. One of the genes residing within $\mathrm{SRO} 2$ is the E-cadherin gene, $C D H 1$. This gene is known to be mutated in lobular breast carcinomas, resulting in loss of E-cadherin expression. However, E-cadherin shows normal expression in most cases of ductal carcinoma, the major mammary cancer type. Thus other genes within 16q22.1 are expected to be involved in the development of this tumor subtype. A minimaltiling path of the contig presented consists of PAC clones, which have the potential of being transferred to mammalian cells as stably replicating episomes. This feature might serve as the basis for a functional strategy in which PACs would be introduced into tumor cells for the identification, verification and characterization of the tumor suppressor gene expected to be present within SRO2.
Fruehauf, John

[60]

\section{New approaches to antiangiogenesis therapy of solid tumors}

John Fruehauf, Eugene Mechetner, Shubhra Mittal, Bejan Shabharhami, Deborah Pavich, Lilian Tee \& Carina Torres

Oncotech, Inc., 1791 Kaiser Avenue, Irvine, California 92614, USA

Anticancer chemotherapy has produced only modest gains in the treatment of solid tumors. Anti-angiogenesis therapy on the basis of rationally designed therapies targeting the tumor vasculature is a new approach currently being pursued in clinical trials. This study is directed at the development of in vitro and in vivo strategies to measure the potential efficacy of anti-angiogenesis compounds. To characterize endothelial cells derived from malignant specimens, we have developed cell separation methods (flow sorting, magnetic immunobeads) to obtain selectively highly purified populations of resting $\left(\mathrm{CD} 105^{-} \mathrm{CD} 31^{+} \mathrm{CD} 45^{-}\right)$versus activated $\left(\mathrm{CD} 105^{+} \mathrm{CD} 31^{+} \mathrm{CD} 45^{-}\right)$endothelial cells. We assessed the functional characteristics of sorted endothelial cells in type I collagen and fibronectin cultures using flow cytometry immunostaining for CD31 and CD105, as well as Aclow-density lipoprotein uptake monitored by fluoresence microscopy as a signature of vascular endothelial cells. We evaluated tubulogenesis in MATRIGEL-based cultures by phase contrast light microscopy. Docitaxel and thalidomide were employed as validation compounds to evaluate differential effects of drug exposure on morphology, cell-surface biomarker expression and apoptotic potential in HUVEC and HAEE1 endothelial cells grown on type I collagen. Annexin V binding was the apoptosis endpoint used for the flow cytometry assay. Research Genetics array technology was used to evaluate gene expression profiles in endothelial cells sorted on the basis of CD31 and CD105 expression or Annexin V binding and expanded in collagen I cultures.

Fung, Eric

[61]

\section{Ciphergen ProteinChip technology: A platform for protein profiling and biomarker identification}

\section{Eric Fung}

Ciphergen Biosystems, 6611 Dumbarton Circle, Fremont, California 94555, USA

ProteinChip proteomics technology can accelerate the discovery of protein biomarkers, including tumor markers. ProteinChip technology incorporates surface-enhanced laser desorption/ionization with mass spectrometry to allow for the rapid profiling and comparison of protein expression in normal and diseased tissues. I present examples of the use of ProteinChip technology to identify new markers of a variety of neoplasms, including bladder cancer, prostate cancer and leukemia. Efforts to identify and validate these biomarkers are under way. I also show that a biomarker panel has increased diagnostic sensitivity and specificity compared with a single biomarker. 